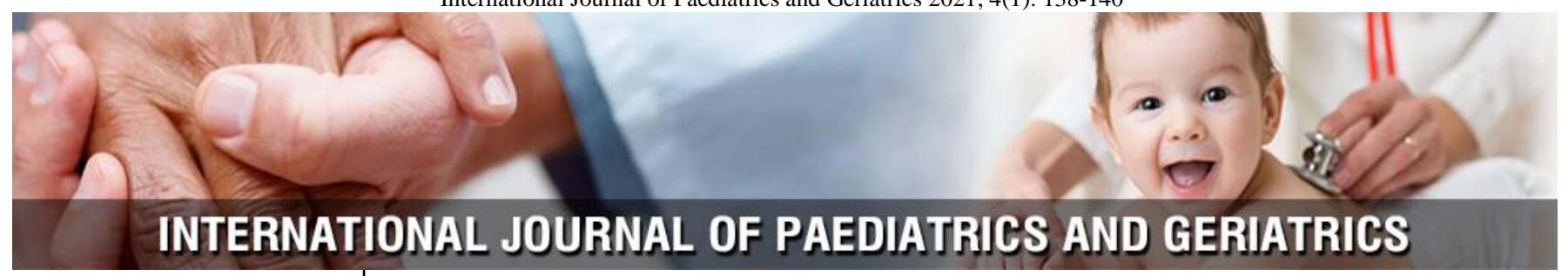

P-ISSN: 2664-3685

E-ISSN: 2664-3693

www.paediatricjournal.com IJPG 2021; 4(1): 138-140

Received: 13-12-2020

Accepted: 16-01-2021

Dr. Rajesh Kumar Singh

Senior Consultant,

Department of TB and

CHEST District Hospital,

Ghazipur, Uttar Pradesh,

India

Dr. Umesh Kumar

Senior Consultant,

Department of Pediatric,

Hospital, Ghazipur, Uttar

Pradesh, India
Corresponding Author: Dr. Rajesh Kumar Singh Senior Consultant, Department of TB and CHEST District Hospital, Ghazipur, Uttar Pradesh, India

\section{Efficacy of Rifapentine plus isoniazid in the treatment of tuberculosis infection in children in India}

\author{
Dr. Rajesh Kumar Singh and Dr. Umesh Kumar
}

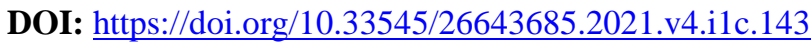

\begin{abstract}
Background: Treatment of tuberculosis (TB) infection effectively prevents its progression and cures it. Persons aged 12 and older can safely and effectively take rifapentine and isoniazid for three months to treat latent tuberculosis infection. Published data for children is limited. Data on the 3-month weekly rifapentine plus isoniazid (3 HP) in children in India are currently unavailable.

Objective: To compare the treatment completion rates and incidence rates of adverse drug reactions among childrens with TB undergoing the $9 \mathrm{H}$ and $3 \mathrm{HP}$ regimens.

Methods: This was a prospective, open-label, multicentre randomised trial involving children with newly diagnosed TB. 263 participants were randomised into two groups to have 131 patients in the $3 \mathrm{HP}$ group and 132 to the $9 \mathrm{H}$ group. Primary endpoint was completion of the 270-day treatment within 12 months. Adverse drug reaction were analysed in both the groups.

Results: Comparing the $9 \mathrm{H}$ group with the $3 \mathrm{HP}$ group, those who dropped out of the $9 \mathrm{H}$ group stopped participating more often. Reason for dropping out was consent withdrawal. Incidence of hepatotoxicity was lower in the $3 \mathrm{HP}$ group than in the $9 \mathrm{H}$ group. However, grade 2 and $3 \mathrm{ADR}$ were more in the $3 \mathrm{HP}$ group.
\end{abstract}

Conclusion: An increased completion rate was seen with the 3HP regimen compared with the $9 \mathrm{H}$ regimen, as well as better tolerance to adverse effects.

Keywords: Children, tuberculosis, isoniazid, rifapentin, adverse drug reaction

\section{Introduction}

One quarter of the world's population has latent tuberculosis infection, and worldwide there are 10 million cases of the disease each year ${ }^{[1]}$. A deadly communicable disease, tuberculosis (TB), still causes deaths ${ }^{[2]}$. The World Health Organization (WHO) aimed to reduce TB-related deaths by $95 \%$ and incidence rate of TB by $90 \%$ by $2035^{[3]}$. Therefore, case finding should be more intensive, as well as preventive treatment introduced to people with latent tuberculosis (LTBI), who carry a 10\% lifetime risk of developing active tuberculosis ${ }^{[3]}$.

Only half of people with multidrug-resistant tuberculosis (MDR) and only $30 \%$ of people with significantly drug-resistant tuberculosis can be cured by WHO-recommended treatments ${ }^{[4]}$. Drug resistance and drug-sensitive tuberculosis can be effectively treated and reduced with the use of new, more potent drugs ${ }^{[4]}$. As a result of collective efforts by stakeholders, advocates, and researchers, shorter courses, safer treatments, and better tolerated treatments are slowly becoming available ${ }^{[5]}$. The development of host-directed therapies (HDTs) is an important adjunct to drug treatment to reduce the duration of treatment, eliminate Mycobacterium tuberculosis infection, reduce persistent lung swelling and prevent drug resistance development. Drug-resistance tuberculosis and ITB-HIV coinfection patients whose survival rates can be improved by treatment regimens ${ }^{[6]}$.

Studies demonstrated that treatment with isoniazid reduced the risk by approximately $60 \%$ in HIV-negative individuals at a high risk of active TB for more than 2 years ${ }^{[7]}$. However, long term isoniazid treatment causes hepatotoxicity ${ }^{[8]}$. The newer short-course TB prevention therapy is based on rifapentine. When combined with a second TB drug, isoniazid, rifapentine forms the 3HP regimen (taken once weekly for 12 weeks) and the 1HP regimen (taken once a day for one month). The 3HP and 1HP regimens offer shorter alternatives to the older standard of care, called isoniazid preventive therapy (IPT), in which people take isoniazid every day for between six and 36 months. 
It has been used to reduce hepatotoxic isoniazid exposure and to increase treatment completion rates by reducing the duration of treatment, the exposure to rifampicin monotherapy and rifampicin-combination therapy ${ }^{[9]}$. In a previously conducted randomized trial, 3-month regimen of weekly rifapentine plus isoniazid (3HP regimen) have exhibited higher completion rates and lower liver toxicity than the 9-month regimen of daily isoniazid (9H regimen) [8].

The incidence of drug-resistant tuberculosis in India is high. There are many variations in its patterns and prevalence across time, region, and setting. Assuring programmatic success requires universal drug susceptibility testing and continuous DR-TB surveillance in all districts. Thus, in this prospective randomised study, we compared the treatment completion rates and incidence rates of adverse drug reactions among childrens with $\mathrm{TB}$ undergoing the $9 \mathrm{H}$ and $3 \mathrm{HP}$ regimens.

\section{Materials and Methods Study design and Subjects}

This was a prospective, open-label, multicentre randomised trial involving childrens with a new diagnosis of pulmonary TB with positive acid-fast bacilli (AFB). The study was approved and conducted in two hospitals. All participants were aged 8-18 years old, close contacts of patients with AFB-positive pulmonary TB and a positive tuberculin skin test (TST) within 1 month of unprotected exposure to be eligible for enrollment. A positive TST was defined as an induration of $\geq 10 \mathrm{~mm}$ read at $48-72 \mathrm{~h}$ after inoculation and an unprotected exposure as exposure duration of $\geq 8 \mathrm{~h}$ in a single day or a cumulative duration of $\geq 40 \mathrm{~h}$ without adequate personal protective equipments for preventing airborne disease transmission. This study excluded the participants if their index patients had negative sputum cultures for Mycobacterium tuberculosis complex, though smear-positive for AFB, or M. tuberculosis complex with resistance to isoniazid or rifampin and those who were suspected to have active pulmonary TB because of clinical symptoms or image examinations, concurrently using drugs with severe drug-drug interactions with or allergic to isoniazid, rifampin, or rifapentine.

\section{Randomisation and follow-up}

During the study period, 290 TST-positive close contacts of AFBpositive TB patients were screened for eligibility; however, 25 refused to participate and 12 had screen failure. Thus, 263 participants were eligible for enrolment. These patients were randomised into two groups to have 131 patients in the $3 \mathrm{HP}$ group and 132 to the $9 \mathrm{H}$ group.

Each participant was assessed at day 1 (baseline), week 4, week 8 , week 12 , week 24 , and week 36 until week 36 in the $9 \mathrm{H}$ group. After completion of treatment, all participants were followed for 2 years after termination, or until active TB developed. The Mycobacterial culture of two sputum specimens was sent if respiratory symptoms existed. The $9 \mathrm{H}$ group had blood tests every month for three months and every two months thereafter as needed to monitor their blood count and liver function. In order to check the adherence of the participants, research assistants and government-paid supporters implemented the DOT method. Inquiries about adverse reactions were made to all participants, either in person or by telephone, every week after treatment.

\section{Outcomes}

Among the $9 \mathrm{H}$ group, the primary endpoint was completion of the 270-day treatment within 12 months. Among the 3 HP group, the primary endpoint was completion of the 12dose treatment within 3 months. Our intention-to-treat population was analyzed to determine the completion rate. We identified the factors that led to early discontinuation of prevention therapy. As a secondary outcome, incidence data was collected for each study group regarding severe adverse reactions.

\section{Adverse drug reaction}

AST/ALT levels were defined as clinically relevant hepatotoxicity as a 2-fold or greater increase from baseline in AST/ALT levels, 3-fold or greater increase with clinical symptoms, or a total bilirubin level greater than $3 \mathrm{mg} / \mathrm{dL}$. In order to determine the likelihood of adverse drug reactions, the Naranjo algorithm was applied. Scores from 5 to 8 indicate probable ADRs, whereas scores over 9 indicate definite ADRs.

\section{Results}

During the study period, 290 TST-positive close contacts of AFBpositive TB patients were screened for eligibility; however, 25 refused to participate and 12 had screen failure. Thus, 263 participants were eligible for enrolment; 131 were randomised to the $3 \mathrm{HP}$ group and 132 to the $9 \mathrm{H}$ group. Ten participants from each study group were excluded late from analysis, leaving 253 participants available for analysis of the primary endpoint. A comparison of the baseline characteristics of the two study groups is presented in Table 1. Till the mean follow-up durations of 710 days after the end of treatment none of the participants developed active TB.

Comparing the $9 \mathrm{H}$ group with the $3 \mathrm{HP}$ group, those who dropped out of the $9 \mathrm{H}$ group stopped participating more often $(23.48 \%$ vs. $9.92 \%, p<0.01)$. Reason for dropping out were consent withdrawal (2.29\% vs. $9.84 \%, p<0.001)$.

$9 \mathrm{H}$ participants had higher total bilirubin, AST and ALT levels than their $3 \mathrm{HP}$ counterparts after 3 months of treatment. Overall, only four patients $(3 \%)$ in the $3 \mathrm{HP}$ group had clinically relevant hepatotoxicity, but eight $(6 \%$, $p<0.05$ ) patients had clinically relevant hepatotoxicity in the $9 \mathrm{H}$ group.

Table 1: Events of drug completion and discontinuation

\begin{tabular}{|c|c|c|}
\hline Variables & 3HP $(\mathbf{n}=\mathbf{1 3 1})$ & $\mathbf{9 H}(\mathbf{n}=\mathbf{1 3 2})$ \\
\hline Non-completion, $\mathbf{n}(\boldsymbol{\%})$ & $13(9.92)$ & $31(23.48)$ \\
\hline Adverse drug reaction & $10(7.63)$ & $18(13.63)$ \\
\hline Consent withdrawal & $3(2.29)$ & $13(9.84)$ \\
\hline Poor adherence & 1 & 8 \\
\hline Prevention not needed & 1 & 3 \\
\hline Being afraid of side effects & 1 & 2 \\
\hline
\end{tabular}

During treatment, ADRs other than hepatotoxicity developed in $45(34.35 \%)$ participants in the $3 \mathrm{HP}$ group and in $30(22.9 \%)$ participants in the $9 \mathrm{H}$ group $(\mathrm{p}>0.05)$. $3 \mathrm{HP}$ groups had significantly more Grade 2 toxicities than $9 \mathrm{H}$ groups $(10.5 \%$ vs. $3.3 \%, p<0.01)$. Grade 3 toxicity occurred in three $(2.29 \%)$ and $1(0.76 \%)$ participant in the $3 \mathrm{HP}$ and $9 \mathrm{H}$ groups, respectively $(\mathrm{p}>0.05$ ). Grade 4 toxicity was not observed in any of the groups.

Ten patients from the $3 \mathrm{HP}$ and 8 from the $9 \mathrm{H}$ group have discontinued from the trial due to various reasons such as 
Flu-like symptoms, especially fever. Treatment terminations were most often caused by hepatitis.

\section{Discussion}

The study showed that the 3HP group had a higher treatment completion rate than the $9 \mathrm{H}$ group, and that they were more likely (but not statistically significant) to discontinue the study drugs due to adverse reactions. ADRs other than hepatotoxicity was more common in the $3 \mathrm{HP}$ group except for clinically relevant hepatotoxicity. No Grade 4 reactions occurred in the 3 HP group despite a higher discontinuation rate due to ADRs than in the $9 \mathrm{H}$ group. Further, ADRs tend to be self-limited and resolve naturally after symptomatic treatment or spontaneously.

The use of 3 HP has been evaluated in various settings including randomised clinical trials [10], observational cohorts ${ }^{[11]}$, retrospective studies ${ }^{[12]}$, and clinics ${ }^{[13]}$. As a whole, the completion rate $(65 \%-100 \%)$ is excellent. This can be explained by the short treatment duration and selflimiting adverse reactions associated with $3 \mathrm{HP}$. In spite of using varying study designs, definitions of hepatotoxicity, monitoring frequencies, and patient populations, studies have discovered rates of hepatotoxicity in patients who receive the $3 \mathrm{HP}$ regimen ranging from $0 \%-1.5 \%$, whereas the rates in patients who receive the $9 \mathrm{H}$ regimen are relatively high $(1.2 \%-5.3 \%)$.

In an Indian population with intermediate numbers of TB, the study was the first prospective trial to compare the completion rates of $3 \mathrm{HP}$ and $9 \mathrm{H}$ regimens. In India, the continent with the highest burden of $\mathrm{TB}$, the findings provided important information on managing LTBI in all ethnicities. Several limitations of this study deserve further discussion. First, the effectiveness of LTBI treatment can't be evaluated due to the short follow-up period. Second, due to the small sample size, we were not able to examine independent risk factors.

\section{Conclusion}

In addition to the literature, our results provide pertinent information about the use of $3 \mathrm{HP}$ in the Indian children. There are no ADRs related to 3HP that are as serious as those related to $9 \mathrm{H}$. However, $3 \mathrm{HP}$ treatment completion rates are higher and rates of clinically relevant hepatotoxicity are lower than those related to $9 \mathrm{H}$ treatment. Although these adverse drug reactions are usually selflimited, they are manageable. LTBI can be treated with rifapentine and isoniazid for 3 months instead of using it daily for 9 months.

\section{References}

1. Suárez I, Fünger SM, Kröger S, Rademacher J, Fätkenheuer G, Rybniker J. The Diagnosis and Treatment of Tuberculosis. Dtsch Arztebl Int. 2019;116(43):729-735.

2. WHO. Annual TB report 2017 Available at: http://www.who.int/iris/handle/10665/

3. 254762, Accessed date: 19 July 2020.

4. World Health Organization 67th World Health Assembly. Global strategy and targets for tuberculosis prevention, care and control after A67/11 Geneva: World Health Organization; 2015 Available at: http://apps.who.int/gb/ebwha/pdf_files/WHA67/A67_1 1-en.pdf.

5. Small PM, Fujiwara PI. Management of tuberculosis in the United States. N Engl J Med. 2001;345:189-200

6. Tiberi S, du Plessis N, Walz G, Vjecha MJ, Rao M, Ntoumi F. Tuberculosis: progress and advances in development of new drugs, treatment regimens, and host-directed therapies. Lancet Infect Dis 2018.

7. Wallis RS, Maeurer M, Mwaba P, et al. Tuberculosisadvances in development of new drugs, treatment regimens, host-directed therapies and biomarkers. Lancet Infect Dis 2016;16:e34-46.

8. Smieja MJ, Marchetti CA, Cook DJ, Smaill FM. Isoniazid for preventing tuberculosis in non-HIV infected persons. Cochrane Database Syst Rev 2000:CD001363.

9. Sterling TR, Villarino ME, Borisov AS, et al. Three months of rifapentine and isoniazid for latent tuberculosis infection. N Engl J Med 2011;365:215566.

10. Kahwati LC, Feltner C, Halpern M, et al. Primary care screening and treatment for latent tuberculosis infection in adults: evidence report and systematic review for the US preventive services task force. J Am Med Assoc 2016;316:970-83.

11. Martinson NA, Barnes GL, Moulton LH, et al. New regimens to prevent tuberculosis in adults with HIV infection N Engl J Med, 2011;365:11-20.

12. Huang YW, Yang SF, Yeh YP, Tsao TC, Tsao SM. Impacts of 12-dose regimen for latent tuberculosis infection: treatment completion rate and costeffectiveness in Taiwan. Medicine (Baltim), 2016;95:e4126.

13. Perez AE, Seo SK, Schneider WJ, Eisenstein C, Brown AE. Management of latent tuberculosis infection among healthcare workers: 10-year experience at a single center. Clin Infect Dis, 2017;65:2105-2111

14. McClintock AH, Eastment M, McKinney CM, et al. Treatment completion for latent tuberculosis infection: a retrospective cohort study comparing 9 months of isoniazid, 4 months of rifampin and 3 months of isoniazid and rifapentine. BMC Infect Dis, 2017; 17:146. 